\title{
Controlling a Cold Store Temperature Through a Mobile Phone
}

\author{
Abdelrasoul Jabar Alzubaidi, Isam muhydeen 2 \\ 1 Electronics Dept-Engineering College-Sudan University of science and Technology \\ Rasoul46@live.com \\ 2 Sudan Academy of Sciences (SAS); Council of Engineering Researches \& Industrial Technologies \\ essammuhiuddin@gmail.com
}

\begin{abstract}
Mobile phone has become familiar and necessary to everyone, and so become the remote control. Therefore, it would be wonderful to join these small devices in one function i.e. to use the mobile phone as a remote control.

Here, a mobile phone is used to serve as a remote control for a cold-store. The remote control system consists of two mobile phones, a dual tone multiple frequency decoder (DTMF), a personal computer (PC), a cold store, and a designed circuit. One mobile phone serves as a remote control, while the other is used to receive calls from the first one. When a call is received the tone is decoded by a DTMF decoder. The decoded signal is used to control the operations in the cold store. This remote control system can be used for any electronic or electrical device to operate it from anywhere using a mobile phone, wherever the mobile network is available.
\end{abstract}

Keywords: - control system, PC, Mobile phone , DTMF, cold store.

\section{INTRODUCTION}

Systems are designed to perform different tasks according to the required functions. For executing such functions, the system has to be easy to operate, well designed and controlled. The control process can be carried out inside the system or remotely. The remote control process can be easily classified into: first, wired control that uses compatible media such as metallic cable or fiber optics. Second, wireless remote control that uses Infra-Red (IR) technology, Ultrasonic (for short ranges), and cellular mobile technologies. The research is made use of cellular mobile technology to give flexibility to the system to be controlled remotely because of the wide spreading of technologies and the possibility of emerging such technology with other due to rapid growing of the applications. The exploit of existing wireless mobile networks in different applications normally leads to the systems emerging. This approach of remote control process improves the control system for better operation and performance to meet the recent requirements within reasonable limit of low economical cost and effective impact.

\section{APPROACH}

Analysis and design are the two principal phases, which are integrated together to build the circuit. Accordingly, analysis should be taken as a first step for evaluating the system and specifying the new requirements of each functional area of the devices that must be controlled. First of all, it is necessary to put all the general required points as main supports to depend on:

- Analysis.

- Determining the system behavior.

- Specifying the functional areas

- Building preliminary diagram as first phase of design.

- Checking the overall diagram

- Testing the designed system.

- Modifications.

Analysis is highly important phase to be firstly carried out in the system as it parts the system into subgroups. Analysis also consists of examining the requirements of subgroups to be very clear.

According to the analysis procedures, the system behavior will be obvious, and shows the logical sequences of the control process. The logical sequences are being done according to the devices priority to show the consistency of devices in performing the operating process.

All the subgroups which reflect the common characteristic and logical sequences are going to yield various functions that the system performs. Then the functional area of each device can be perceived easily and taken into account in the design phase. The more important thing in this phase is the well-specified overlapped functional areas in order to be well designed. 
Then all the above points will show the main features of the developed system, and consequently it is easy to build preliminary diagram of the proposed design as shown in figure (1) for determining the following operations sequence :

- Remote control of the power supply activation or shut down.

- Compressor operation.

- Two minutes delay.

- Evaporator operation.

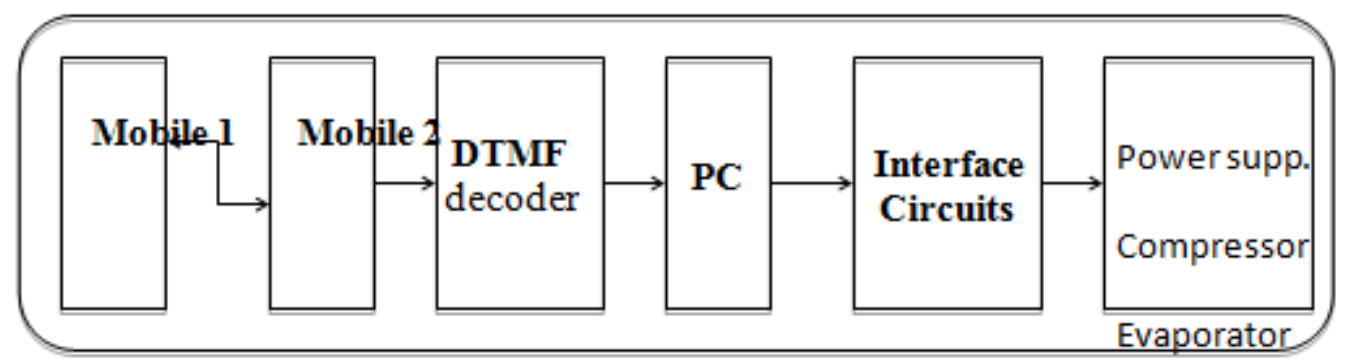

Figure (1) block diagram of the system

After that, the preliminary diagram should be checked to ensure that all the overlapped functional areas and the logical sequences of control signal are determined and specified in the right way.

Testing phase is also very important to be made for all devices and functional areas to reflect devices role in system. The test can be grouped into following:

- Integrating test: to show how all the two different technologies (Wireless cellular mobile communications and computer network) are well-interfaced to each other.

- Acceptance test: shows that the overall system performs according to the requirements.

Modifications process should be done to correct any mistakes that might take place in any phase . Modifications process is made for the improvements of the system to meet all the new requirements.

\section{SYSTEM COMPONENTS}

\section{DTMF Decoder}

DTMF is short for Dual Tone Multi Frequency. It is a generic communication term for touch tone (a Registered Trademark of AT\&T). The tones produced when dialing on the keypad on the phone could be used to represent the digits, and a separate tone is used for each digit. Pressing any key generate unique tone which consists of two different frequencies one each of higher and lower frequency range. The resultant tone is convolution of two frequencies. Figure 2 shows mobile keypad frequencies and table1 also shows tone frequency associated with a particular key.

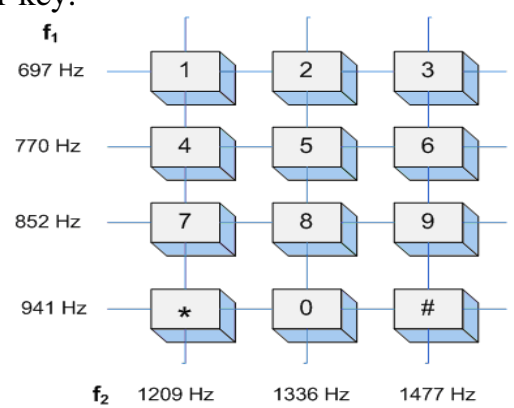

Figure 2 Phone keypad for DTMF generation [10]

Each of these tones is composed of two pure sine waves of the low and high frequencies superimposed on each other. These two frequencies explicitly represent one of the digits on the telephone keypad. Thus generated signal can be expressed mathematically as follows:

Where;

$$
f(t)=A H \sin (2 \pi f H t)+A L \sin (2 \pi f L t)
$$

$\mathrm{AH}, \mathrm{AL}$ are the amplitudes.

$\mathrm{fH}, \mathrm{fL}$ are the frequencies of high \& low frequency range.

The properties of DTMF tone frequencies are:

- No frequency is an integer multiple of another.

- The difference between any two frequencies does not equal any of the frequencies 
- The sum of any two frequencies does not equal any of the frequencies

\section{PC Computer:}

PC computer hosts developed software using $\mathrm{C}++$ programming language to control cutting machine. The PC computer is connected with 8870 DTMF decoder via parallel port inputs. The software dictates the processor to handle controlling process. A corresponding signal is then sent via the output pins of the parallel port to the HD74LS373 latch IC.

\section{HD74LS373 Latching IC:}

The HD74LS373 is eight bit is register IO mapped used as a buffer which stores signals for future use. Different types of latches are available HD74LS373 octal D-type transparent latch will be used in this system. This type of latch is suitable for driving high capacitive and impedance loads.

\section{ULN 2001A Darlington IC:}

The ULN2803A is a high-voltage, high-current Darlington transistor array. The device consists of eight NPN Darlington pairs that feature high-voltage outputs with common-cathode clamp diodes for switching inductive loads. The collector-current rating of each Darlington pair is $500 \mathrm{~mA}$. The Darlington pairs may be connected in parallel for higher current capability.

5. cold store :

The cold store is a chamber used for storage of food products. It is supplied with an a manual control panel.

\section{ALGORITHM}

The proposed computer algorithm includes a strict sequence of steps for the operation of the cold store . Similarly reverse steps are to be conducted to shut down the cold store .The algorithm is ;

Start

--- Check the incoming mobile dial for authorization.

- If authorized, continue processing.

- If not authorized, deny access and wait for a mobile dial.

--- Clear all controlled devices .The DTMF code is (0000)2 .

--- Analyze the incoming code from the DTMF decoder.

--- If the code is equal to (0001)2, then activate the power supply of the cold store.

--- If the code is equal to (0010) 2 , then activate the processor of the cold store .

--- If the code is equal to (0011)2, then generate a two minutes delay .

--- If the code is equal to $(0100) 2$, then activate the evaporator of the cold store .

--- If the code is equal to (0101)2, then put off the evaporator of the cold store .

--- If the code is equal to (0110) 2 , then put off the processor of the cold store.

--- If the code is equal to (0111)2, then put off the power supply of the cold store.

End.

\section{RESULTS}

Following are the results are from implementing the proposed system.

a. Results of Control commands in the system with correspondent devices:

Table 1 System commands and their correspondent action on the devices

\begin{tabular}{|l|l|l|l|}
\hline Pressed key & Command & Device & Result \\
\hline 0 & Start & Clear all devices & initialization \\
\hline 1 & Activate power & Power supply & Step1 \\
\hline 2 & Compressor ON & Compressor & Step2 \\
\hline 3 & delay & ------------ & Delay generation \\
\hline 4 & Evaporator ON & Evaporator & Step4 \\
\hline 5 & Evaporator OFF & Evaporator & Step5 \\
\hline 6 & Compressor OFF & Compressor & Step6 \\
\hline 7 & Deactivate power & Power supply & Step7 \\
\hline & & & \\
\hline & & & \\
\hline
\end{tabular}




\section{CONCLUSION}

A remote control is an electronic device used for the remote operation of a machine. The designed circuit make use of radio frequency to transmit the control signal and hence it can be used for control from almost anywhere at any time once the mobile phone ( GSM ,CDMA, etc) network is available.

Using mobile phone as a remote control, we can control any devices before remotely.

Security is essential to ensure that the system is accessible by the authorized personnel only. Non authorized commands to the system will be denied.

\section{REFERANCES}

[1] Manual on meat cold store operation and management By/Dr G. Cano-Muz ApriI.202008 http://www.fao.org/DOCREP/OO4/TOO98E/TOO98EOO.HTM

[2] How Cell Phones Work April.22.2008 By/Julia Layton, Marshall Brain and Jeff Tyson http://www. howstuffworks.com/ceIl—phone. htm

[3] Remote control http://en.wikipedia.org/wiki/Remotecontrol April.1O.2008.

[4] Radio control httpengineeringtalk.com/ March. 15.2008

[5] Remote control by phone http://apogeekits.com March.20.2008

[6] History of remote control http://www.modellbah nottcom/tqpage/ihistory.html March.5.2008 